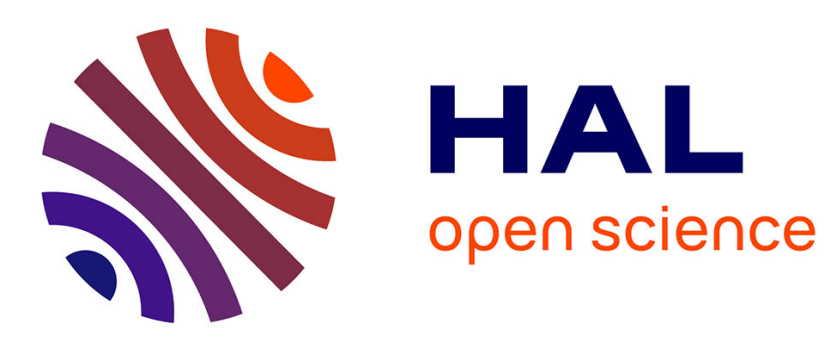

\title{
SOME VARIANTS OF ORPONEN'S THEOREM ON VISIBLE PARTS OF FRACTAL SETS
}

\author{
Carlos Matheus
}

\section{To cite this version:}

Carlos Matheus. SOME VARIANTS OF ORPONEN'S THEOREM ON VISIBLE PARTS OF FRACTAL SETS. 2020. hal-02500421

\section{HAL Id: hal-02500421 \\ https://hal.science/hal-02500421}

Preprint submitted on 5 Mar 2020

HAL is a multi-disciplinary open access archive for the deposit and dissemination of scientific research documents, whether they are published or not. The documents may come from teaching and research institutions in France or abroad, or from public or private research centers.
L'archive ouverte pluridisciplinaire HAL, est destinée au dépôt et à la diffusion de documents scientifiques de niveau recherche, publiés ou non, émanant des établissements d'enseignement et de recherche français ou étrangers, des laboratoires publics ou privés. 


\title{
SOME VARIANTS OF ORPONEN'S THEOREM ON VISIBLE PARTS OF FRACTAL SETS
}

\author{
CARLOS MATHEUS
}

\begin{abstract}
It was recently established by $\mathrm{T}$. Orponen that the visible parts from almost every direction of a compact subset of $\mathbb{R}^{n}$ have Hausdorff dimension at most $n-\frac{1}{50 n}$.

In this note, we refine Orponen's argument in order to show that the visible parts from almost every direction of a compact subset of $\mathbb{R}^{n}$ have Hausdorff dimension at most $n-\min \left\{\frac{1}{5}, \frac{1}{n+2}\right\}$.

Moreover, we also show that some classes of dynamically defined Cantor sets $K \subset \mathbb{R}^{n}$ with Hausdorff dimension $d>\max \left\{\sqrt{3}, \frac{(n-1)+\sqrt{(n-1)(n+3)}}{2}\right\}$ have visible parts of Hausdorff dimension at $\operatorname{most} \max \left\{\frac{3 d+3}{d+3}, \frac{(n+1) d+(n-1)}{d+2}\right\}$ from almost every direction.
\end{abstract}

\section{INTRODUCTION}

Let $K$ be a compact subset of the Euclidean space $\mathbb{R}^{n}, n \geq 2$. Intuitively, the visible part $\operatorname{Vis}_{e}(K)$ of $K$ in the direction $e \in S^{n-1}$ is the subset of $K$ consisting of the points which are first hit by a light beam travelling in the direction $e$ emanating from a certain affine hyperplane orthogonal to $e$.

More concretely, if $\pi_{e}: \mathbb{R}^{n} \rightarrow e^{\perp}$ denotes the orthogonal projection to the hyperplane $e^{\perp}$ orthogonal to $e$ and $\langle.,$.$\rangle stands for the usual Euclidean inner product,$ then $\operatorname{Vis}_{e}(K)$ is the collection of $\leq_{e}$-minimal points of $K$ where $\leq_{e}$ is the partial order defined by $x \leq_{e} y$ if and only if $\pi_{e}(x)=\pi_{e}(y)$ and $\langle x, e\rangle \leq\langle y, e\rangle$.

In general, the visible parts $\operatorname{Vis}_{e}(K)$ are Borel sets because they are the graphs of lower semi-continuous functions, cf. [4, Remark 2.2 (a)].

By definition, $\pi_{e}\left(\operatorname{Vis}_{e}(K)\right)=\pi_{e}(K)$ for all $e \in S^{n-1}$. Therefore, Mattila's extension of Marstrand's theorem [7] provides the following lower bound on the Hausdorff dimension of typical visible parts:

$$
\operatorname{dim}_{H}\left(\operatorname{Vis}_{e}(K)\right) \geq \min \left\{\operatorname{dim}_{H}(K), n-1\right\}
$$

for Lebesgue almost every $e \in S^{n-1}$.

The visibility conjecture asserts that the converse inequality is true, i.e., if $\operatorname{dim}_{H}(K)>n-1$, then $\operatorname{dim}_{H}\left(\operatorname{Vis}_{e}(K)\right)=n-1$ for Lebesgue almost every $e \in S^{n-1}$ (see, e.g., [8, Problem 11]).

It is known that this conjecture admits a positive answer for several particular classes of compact subsets of $\mathbb{R}^{n}$ (cf. [4], [2] and [1]). Furthermore, we know that if $K \subset \mathbb{R}^{n}$ is a compact subset with $d$-Hausdorff measure $0<\mathcal{H}^{d}(K)<\infty$, then the $d$-Hausdorff measure of $\operatorname{Vis}_{e}(K)$ is zero for Lebesgue almost every $e \in S^{n-1}$ (see [5, Theorem 1.1]).

More recently, T. Orponen [9] obtained an unconditional estimate on the Hausdorff dimension of typical visible parts of compact subsets $K$ of $\mathbb{R}^{n}$ : in a nutshell, he proved that $\operatorname{dim}_{H}\left(\operatorname{Vis}_{e}(K)\right) \leq n-\frac{1}{50 n}$ for Lebesgue almost every $e \in S^{n-1}$.

In this note, we refine Orponen's methods to establish the following two results:

Date: March 5, 2020. 
Theorem 1.1. Let $K \subset \mathbb{R}^{n}$ be a compact subset. Then, for Lebesgue almost every $e \in S^{n-1}$, the Hausdorff dimension of $\operatorname{Vis}_{e}(K)$ is at most $n-\min \left\{\frac{1}{5}, \frac{1}{n+2}\right\}$.

Theorem 1.2. Let $K \subset \mathbb{R}^{n}$ be a product of $C^{2}$-dynamically defined Cantor sets of the real line or a self-similar set defined by a finite collection of Euclidean similarities verifying the open set condition. If the Hausdorff dimension of $K$ is $\operatorname{dim}_{H}(K)>$ $\max \left\{\sqrt{3}, \frac{(n-1)+\sqrt{(n-1)(n+3)}}{2}\right\}$, then, for Lebesgue almost every $e \in S^{n-1}$, the Hausdorff dimension of $\operatorname{Vis}_{e}(K)$ is at most $\max \left\{\frac{3 d+3}{d+3}, \frac{(n+1) d+(n-1)}{d+2}\right\}$.

The remainder of this note is divided into two sections: its first half contains the proof of Theorem 1.1 and its second half is devoted to the proof of Theorem 1.2.

\section{Visible PARTS OF GENERAL COMPACT SUbSETS}

Let $K$ be a compact subset of $\mathbb{R}^{n}, n \geq 2$. Up to rescaling, we can (and do) assume that $K \subset[0,1]^{n}$. Since the conclusion of Theorem 1.1 always holds when $K$ has Hausdorff dimension $\leq n-\min \left\{\frac{1}{5}, \frac{1}{n+2}\right\}$, we can (and do) also assume that

$$
n-\min \left\{\frac{1}{5}, \frac{1}{n+2}\right\}<d:=\operatorname{dim}_{H}(K) \leq n .
$$

2.1. Some preliminaries. Recall that the $s$-dimensional Hausdorff measure at scale $0<\rho \leq \infty$ of a subset $E \subset \mathbb{R}^{n}$ is

$$
\mathcal{H}_{\rho}^{s}(E):=\inf \left\{\sum_{i=1}^{\infty} \operatorname{diam}\left(U_{i}\right)^{s}: E \subset \bigcup_{i \geq 1} U_{i} \text { and } \operatorname{diam}\left(U_{i}\right)<\delta \forall i \geq 1\right\}
$$

and the $s$-dimensional Hausdorff measure of $E$ is $\mathcal{H}^{s}(E)=\lim _{\rho \rightarrow 0} \mathcal{H}_{\rho}^{s}(E)$, so that the Hausdorff dimension of $E$ is

$$
\operatorname{dim}_{H}(E):=\inf \left\{s: \mathcal{H}^{s}(E)=0\right\}=\sup \left\{s: \mathcal{H}^{s}(E)=\infty\right\} .
$$

Recall also that a dyadic cube $Q \subset[0,1]^{n}$ is a cube of the form $Q=\prod_{j=1}^{n}\left[\frac{i_{j}}{2^{N}}, \frac{i_{j}+1}{2^{N}}\right]$ for some $N \in \mathbb{N}$ and $\left(i_{1}, \ldots, i_{n}\right) \in\left\{1, \ldots, 2^{N}-1\right\}^{n}$. In the sequel, the collection of dyadic cubes with sides of fixed size $2^{-N}$ is denoted by $\mathcal{D}_{2^{-N}}$.

In [9, Lemma A.1], Orponen showed the following version of Frostman's lemma:

Lemma 2.1 (Orponen). Let $E \subset[0,1]^{n}$ be a compact subset. Then, there exists a Radon measure $\mu$ supported on $E$ and a constant $0<C=C(n)<\infty$ such that $\mu(B(x, r)) \leq C r^{s}$ for all $x \in \mathbb{R}^{n}, r>0$, and

$$
\mu(Q) \geq C^{-1} \min \left\{\mathcal{H}_{\infty}^{s}(E \cap Q), \mathcal{H}^{n}(Q)\right\}
$$

for all dyadic cube $Q \subset[0,1]^{n}$.

Similarly to Orponen [9], our long-term goal is to apply this lemma to estimate the Hausdorff dimension of visible parts in typical directions.

For this sake, we fix first some rational parameters

$$
\begin{gathered}
n-\min \left\{\frac{1}{5}, \frac{1}{n+2}\right\}<n-\varepsilon_{0}<s_{0}^{\prime \prime}<s_{0}^{\prime}<s_{0}<d \leq n, \\
\alpha:=\min \left\{s_{0}^{\prime \prime}-1,2-\frac{s_{0}^{\prime}-(n-1)}{2}\right\}, \\
\frac{\varepsilon_{0} n}{2}<\frac{\varepsilon_{1}}{2}<\min \left\{s_{0}^{\prime \prime}-1,1\right\}-\frac{\varepsilon_{0} n}{2}-2 \varepsilon_{0},
\end{gathered}
$$


and

$$
0<\varepsilon_{*}<\min \left\{s_{0}^{\prime}+\varepsilon_{0}-n, \frac{2}{3}\left(\min \left\{s_{0}^{\prime \prime}-1,1\right\}-\frac{\varepsilon_{0} n}{2}-2 \varepsilon_{0}-\frac{\varepsilon_{1}}{2}\right)\right\} .
$$

Note that these conditions are mutually compatible: indeed, our assumption (2.1) allows us to choose $s_{0}, s_{0}^{\prime}, s_{0}^{\prime \prime}$ and $\varepsilon_{0}$ in $(2.2)$; since $\varepsilon_{0}<\min \left\{\frac{1}{5}, \frac{1}{n+2}\right\}$ and $s_{0}^{\prime}>$ $n-\varepsilon_{0}$, we can select $\varepsilon_{1}$ in (2.4) and $\varepsilon_{*}$ in (2.5).

Now, we use Lemma 2.1 to get $\mu$ supported on $K$ such that

$$
\mu(B(x, r)) \leq C r^{s_{0}}
$$

for all $x \in \mathbb{R}^{n}$ and $r>0$, and

$$
\mu(Q) \geq C^{-1} \min \left\{\mathcal{H}_{\infty}^{s_{0}}(K \cap Q), \mathcal{H}^{n}(Q)\right\}
$$

for all dyadic cube $Q \subset[0,1]^{n}$ (where $0<C=C(n)<\infty$ is a constant).

Recall that (2.6) implies that the $s_{0}^{\prime}$-energy of $\mu$ is finite, i.e.,

$$
I_{s_{0}^{\prime}}(\mu):=\iint \frac{d \mu(x) d \mu(y)}{|x-y|^{s_{0}^{\prime}}}<\infty .
$$

Remark 2.2. For later reference, let us remind that the s-energy of a measure $\theta$ can be expressed in terms of the Fourier transform as

$$
I_{s}(\theta)=\iint \frac{d \theta(x) d \theta(y)}{|x-y|^{s}}=c_{1}(s, n) \int|\widehat{\theta}(\xi)|^{2} \cdot|\xi|^{s-n} d \mathcal{H}^{n}(\xi)
$$

where $0<c_{1}(s, n)<\infty$ is a constant.

In the sequel, $\delta=2^{-N}, N \in \mathbb{N}$, is an arbitrary (small) dyadic scale such that $\delta^{\varepsilon_{0}}$ is also a dyadic scale.

2.2. Contribution of light cubes. We say that a dyadic cube $Q \in \mathcal{D}_{\delta}$ is $\delta$-light when $\mu(Q) \leq \delta^{n+\varepsilon_{*}}$. The portion of $K$ contained in $\delta$-light cubes is denoted by $K_{\delta \text {,light. Since }} \mathcal{D}_{\delta}$ has cardinality $\delta^{-n}$, it follows from (2.7) that:

Lemma 2.3. $\mathcal{H}_{\infty}^{s_{0}}\left(K_{\delta, l i g h t}\right) \leq C(n) \cdot \delta^{\varepsilon_{*}}$.

In particular, this lemma says that we can safely focus on the $\delta$-heavy portion $K_{\delta \text {,heavy }}:=K \backslash K_{\delta \text {,light }}$ of $K$.

2.3. Exceptional directions. Given a dyadic cube $Q \in \mathcal{D}_{\delta^{\varepsilon_{0}}}$, the restriction of $\mu$ to $Q$ is denoted by $\mu_{Q}$. The set of $\delta$-exceptional directions associated to $Q$ is

$$
E_{\delta, Q}:=\left\{e \in S^{n-1}: \int_{e^{\perp}}\left|\widehat{\mu_{Q}}(\zeta)\right|^{2} \cdot|\zeta|^{s_{0}^{\prime}-(n-1)} d \mathcal{H}^{n-1}(\zeta) \geq \delta^{-\varepsilon_{1}}\right\} .
$$

Since $I_{s_{0}^{\prime}}\left(\mu_{Q}\right) \leq I_{s_{0}^{\prime}}(\mu)$, it follows from (2.8) and a change of variables to polar coordinates in Remark 2.2 that:

Lemma 2.4. $\mathcal{H}^{n-1}\left(E_{\delta, Q}\right) \leq c_{2}\left(s_{0}^{\prime}, n\right) I_{s_{0}^{\prime}}(\mu) \delta^{\varepsilon_{1}}$ for all $Q \in \mathcal{D}_{\delta^{\varepsilon_{0}}}$.

2.4. Good and bad lines. Denote by $\mathcal{L}_{e}$ the space of lines parallel to $e \in S^{n-1}$. Given a dyadic cube $Q \in \mathcal{D}_{\delta^{\varepsilon_{0}}}$ intersecting $K$, the set $\mathcal{L}_{e, \delta \text {,bad, } Q}$ of $\delta$-bad lines in direction $e$ associated to $Q$ consists of all lines $\ell \in \mathcal{L}_{e}$ disjoint from $K \cap Q$ whose $2 \delta$-neighborhood $\ell(2 \delta)$ satisfy

$$
\#\left\{R \in \mathcal{D}_{\delta}: R \subset Q, R \cap K \neq \emptyset, R \text { is not light, } R \cap \ell(2 \delta) \neq \emptyset\right\} \geq \delta^{2 \varepsilon_{0}-1} .
$$


We say that $\ell \in \mathcal{L}_{e}$ is a $\delta$-good line in the direction $e$ whenever $\ell \notin \mathcal{L}_{e, \delta, \text { bad, } Q}$ for all $Q \in \mathcal{D}_{\delta^{\varepsilon_{0}}}$ intersecting $K$. The collection of $\delta$-good lines in the direction $e$ is denoted by $\mathcal{L}_{e, \delta \text {,good }}$ and we define

$$
L_{e, \delta, \text { good }}:=\bigcup_{\ell \in \mathcal{L}_{e, \delta, \text { good }}} \ell .
$$

Lemma 2.5. $\mathcal{H}_{\delta}^{s_{0}^{\prime}}\left(\operatorname{Vis}_{e}(K) \cap K_{\delta, \text { heavy }} \cap L_{e, \delta, \text { good }}\right) \leq \delta^{\varepsilon_{*}}$ for all $\delta$ sufficiently small.

Proof. Let us use a collection $\mathcal{T}_{e, \delta}$ tubes of width $\delta$ whose bases are perpendicular to $e$ in order to cover $[0,1]^{n}$. Since $\# \mathcal{T}_{e, \delta} \leq c_{3}(n) \delta^{-(n-1)}$, our task is reduced to prove that, for each $T \in \mathcal{T}_{e, \delta}$, the minimal number $N\left(\operatorname{Vis}_{e}(K) \cap K_{\delta \text {, heavy }} \cap L_{e, \delta, \text { good }} \cap T, \delta\right)$ of $\delta$-balls needed to cover $\operatorname{Vis}_{e}(K) \cap K_{\delta \text {, heavy }} \cap L_{e, \delta \text {,good }} \cap T$ is at most

$$
N\left(\operatorname{Vis}_{e}(K) \cap K_{\delta, \text { heavy }} \cap L_{e, \delta, \text { good }} \cap T, \delta\right) \leq c_{5}(n) \delta^{\varepsilon_{0}-1} .
$$

Indeed, the estimates above imply that

$$
\mathcal{H}_{\delta}^{s_{0}^{\prime}}\left(\operatorname{Vis}_{e}(K) \cap K_{\delta, \text { heavy }} \cap L_{e, \delta, \text { good }}\right) \leq c_{3}(n) c_{5}(n) \delta^{-(n-1)} \delta^{\varepsilon_{0}-1} \delta^{s_{0}^{\prime}} \leq \delta^{\varepsilon_{*}}
$$

for all $\delta$ sufficiently small thanks to the fact that $s_{0}^{\prime}+\varepsilon_{0}-n>\varepsilon_{*}$ (cf. (2.5)).

In order to estimate $N\left(\operatorname{Vis}_{e}(K) \cap K_{\delta \text {, heavy }} \cap L_{e, \delta \text {,good }} \cap T, \delta\right)$ for a given $T \in \mathcal{T}_{e, \delta}$, we consider two scenarios:

(i) for all $Q \in \mathcal{D}_{\delta^{\varepsilon_{0}}}$ intersecting $K$, one has

$$
\#\left\{R \in \mathcal{D}_{\delta}: R \subset Q, R \cap K \neq \emptyset, R \text { is not light, } R \cap T \neq \emptyset\right\}<\delta^{2 \varepsilon_{0}-1} ;
$$

(ii) there exists $Q_{1} \in \mathcal{D}_{\delta^{\varepsilon_{0}}}$ intersecting $K$ with

$$
\#\left\{R \in \mathcal{D}_{\delta}: R \subset Q_{1}, R \cap K \neq \emptyset, R \text { is not light, } R \cap T \neq \emptyset\right\} \geq \delta^{2 \varepsilon_{0}-1} .
$$

In the first scenario, we have that $N\left(\operatorname{Vis}_{e}(K) \cap K_{\delta \text {, heavy }} \cap L_{e, \delta \text {,good }} \cap T, \delta\right) \leq \delta^{\varepsilon_{0}-1}$ simply because $T$ can meet at most $\delta^{-\varepsilon_{0}}$ dyadic cubes $Q \in \mathcal{D}_{\delta^{\varepsilon_{0}}}$.

In the second scenario, we take $Q_{1}$ to be a $\leq_{e}$-minimal dyadic cube with the property described in (ii) (in the sense that $Q_{1}$ minimizes $\inf \left\{\langle x, e\rangle: x \in Q_{1}\right\}$ among all dyadic cubes in (ii)). Since the $2 \delta$-neighborhood of any line $\ell \subset T$ contains $T$, we also have

$$
\#\left\{R \in \mathcal{D}_{\delta}: R \subset Q_{1}, R \cap K \neq \emptyset, R \text { is not light, } R \cap \ell(2 \delta) \neq \emptyset\right\} \geq \delta^{2 \varepsilon_{0}-1} .
$$

Therefore, it follows from the definition of $\delta$-good line that any $\ell \in \mathcal{L}_{e, \delta \text {,good }}$ included in $T$ must intersect $K \cap Q_{1}$.

We affirm that

$$
\operatorname{Vis}_{e}(K) \cap L_{e, \delta, \text { good }} \cap T \cap Q=\emptyset
$$

for any dyadic cube $Q \in \mathcal{D}_{\delta^{\varepsilon_{0}}}$ with $\inf \{\langle x, e\rangle: x \in Q\}>\sup \left\{\langle y, e\rangle: y \in Q_{1}\right\}$. In fact, if $x \in \operatorname{Vis}_{e}(K) \cap L_{e, \delta \text {,good }} \cap T \cap Q$, then $\pi_{e}(x)=\pi_{e}(y)$ for some $y \in Q_{1}$. Since $\langle x, e\rangle>\langle y, e\rangle$, one would get $x \notin \operatorname{Vis}_{e}(K)$, a contradiction.

Hence, $\operatorname{Vis}_{e}(K) \cap L_{e, \delta \text {,good }} \cap T$ is covered by the collection of dyadic cubes $Q \in$ $\mathcal{D}_{\delta^{\varepsilon_{0}}}$ with $\inf \{\langle x, e\rangle: x \in Q\} \leq \sup \left\{\langle y, e\rangle: y \in Q_{1}\right\}$. Now, we observe that

- the number of dyadic cubes $Q \in \mathcal{D}_{\delta^{\varepsilon_{0}}}$ intersecting $T$ with

$$
\inf \left\{\langle z, e\rangle: z \in Q_{1}\right\} \leq \inf \{\langle x, e\rangle: x \in Q\} \leq \sup \left\{\langle y, e\rangle: y \in Q_{1}\right\}
$$

is bounded by an absolute constant $c_{4}(n)$; for each of them, we will use the crude bound $N\left(\operatorname{Vis}_{e}(K) \cap L_{e, \delta \text {,good }} \cap T, \delta\right) \leq \delta^{\varepsilon_{0}-1}$ coming from the fact that $Q \cap T$ can be covered using at most $\delta^{\varepsilon_{0}-1}$ balls of radius $\delta$; 
- any dyadic cube $Q \in \mathcal{D}_{\delta^{\varepsilon_{0}}}$ intersecting $T \cap K$ with

$$
\inf \left\{\langle z, e\rangle: z \in Q_{1}\right\}>\inf \{\langle x, e\rangle: x \in Q\}
$$

satisfies

$\#\left\{R \in \mathcal{D}_{\delta}: R \subset Q_{1}, R \cap K \neq \emptyset, R\right.$ is not light,$\left.R \cap \ell(2 \delta) \neq \emptyset\right\} \leq \delta^{2 \varepsilon_{0}-1}$

because of the $\leq_{e}$-minimality of $Q_{1}$; the number of such cubes $Q$ is at most $\leq \delta^{-\varepsilon_{0}}$ because $T$ meets at most $\delta^{-\varepsilon_{0}}$ dyadic cubes $Q \in \mathcal{D}_{\delta^{\varepsilon_{0}}}$.

By combining the estimates above, we conclude that

$$
N\left(\operatorname{Vis}_{e}(K) \cap K_{\delta \text {,heavy }} \cap L_{e, \delta \text {,good }} \cap T, \delta\right) \leq c_{4}(n) \delta^{\varepsilon_{0}-1}+\delta^{-\varepsilon_{0}} \delta^{2 \varepsilon_{0}-1}=c_{5}(n) \delta^{\varepsilon_{0}-1} .
$$

This completes the proof.

2.5. Typical visible parts in bad lines. The last step towards the proof of Theorem 1.1 is the following estimate:

Lemma 2.6. Let $Q \in \mathcal{D}_{\delta^{\varepsilon_{0}}}$ be a dyadic cube intersecting $K$, consider a direction $e \notin E_{\delta, Q}$, and denote $L_{e, \delta, b a d, Q}:=\bigcup_{\ell \in \mathcal{L}_{e, \delta, b a d, Q}} \ell$. Then,

$$
\mathcal{H}_{\infty}^{s_{0}^{\prime}-1}\left(\pi_{e}\left(L_{e, \delta, b a d, Q}\right)\right) \leq \delta^{\varepsilon_{*}+\varepsilon_{0} n}
$$

for all $\delta$ sufficiently small.

Proof. By contradiction, suppose that $\mathcal{H}_{\infty}^{s_{0}^{\prime}-1}\left(\pi_{e}\left(L_{e, \delta, \text { bad, } Q}\right)\right) \geq \delta^{\varepsilon_{*}+\varepsilon_{0} n}$. By Orponen's version of Frostman's lemma (cf. Lemma 2.1), we have a probability measure $\nu$ supported on $H_{e, \delta, Q}:=\pi_{e}\left(L_{e, \delta, \text { bad }, Q}\right)$ such that

$$
\nu(B(x, r)) \leq C(n-1) \delta^{-\varepsilon_{*}-\varepsilon_{0} n} r^{s_{0}^{\prime}-1}
$$

for all $x \in H$ and $r>0$. Thus, our choice of $\alpha \leq s_{0}^{\prime \prime}-1<s_{0}^{\prime}-1$ in (2.3) (and Remark 2.2) means that the $\alpha$-energy of $\nu$ satisfies

$$
c_{1}(\alpha, n-1) \int|\widehat{\nu}(\xi)|^{2} \cdot|\xi|^{\alpha} d \xi=I_{\alpha}(\nu) \leq c_{6}\left(s_{0}^{\prime \prime}, s_{0}^{\prime}, n\right) \delta^{-\varepsilon_{*}-\varepsilon_{0} n} .
$$

Next, we observe that, by definition, any line $\ell \in \mathcal{L}_{e, \delta \text {,bad, } Q}$ misses $K \cap Q$. Therefore, $\mu_{Q, e}:=\left(\pi_{e}\right)_{*}\left(\mu_{Q}\right)$ and $\nu$ have disjoint supports. Hence, if we fix a non-negative smooth bump function $\varphi$ on $e^{\perp} \simeq \mathbb{R}^{n-1}$ with total integral one and $\varphi(0)=1$, then

$$
\begin{aligned}
0 & =\int \varphi_{\eta} * \mu_{Q, e} d \nu=\int \widehat{\varphi}(\eta \xi) \widehat{\mu_{Q, e}}(\xi) \overline{\widehat{\nu}(\xi)} d \xi \\
& =\int\left(1-\widehat{\varphi}\left(c_{7}(n) \delta \xi\right)\right) \widehat{\varphi}(\eta \xi) \widehat{\mu_{Q, e}}(\xi) \overline{\widehat{\nu}(\xi)} d \xi+\int \widehat{\varphi}\left(c_{7}(n) \delta \xi\right) \widehat{\varphi}(\eta \xi) \widehat{\mu_{Q, e}}(\xi) \overline{\widehat{\nu}(\xi)} d \xi \\
& :=A_{2}-A_{1}
\end{aligned}
$$

for all $0<\eta \ll \delta$, where $\varphi_{\eta}(x)=\varphi(\eta x) / \eta^{n-1}$.

In the sequel, we will reach a contradiction with the identity in the previous paragraph by showing that $\left|A_{2}\right|<\left|A_{1}\right|$. For this sake, we observe that $\widehat{\varphi}$ is a bounded Lipschitz function with $\widehat{\varphi}(0)=1$, so that $\left|1-\widehat{\varphi}\left(c_{7}(n) \delta \xi\right)\right| \leq c_{8}(n) \delta|\xi|$ and, a fortiori,

$\left|A_{2}\right| \leq c_{8}(n) \delta^{\frac{s_{0}^{\prime}-(n-1)}{2}+\frac{\alpha}{2}}\left(\int\left|\widehat{\mu_{Q, e}}(\xi)\right|^{2} \cdot|\xi|^{s_{0}^{\prime}-(n-1)} d \xi\right)^{1 / 2}\left(\int|\widehat{\nu}(\xi)|^{2} \cdot|\xi|^{\alpha} d \xi\right)^{1 / 2}$

thanks to our choice of $\frac{s_{0}^{\prime}-(n-1)}{2}+\frac{\alpha}{2} \leq 1$ in (2.3) and the Cauchy-Schwarz inequality. By plugging into the previous inequality the facts that our choices in (2.2) and (2.3) imply $\frac{s_{0}^{\prime}-(n-1)}{2}+\frac{\alpha}{2} \geq \min \left\{s_{0}^{\prime \prime}-1,1\right\}$, our assumption $e \notin E_{\delta, Q}$ allows (by definition) 
to control $\left|\widehat{\mu_{Q}}(\xi)\right|\left(=\left|\widehat{\mu_{Q, e}}(\xi)\right|\right.$ for $\left.\xi \in e^{\perp}\right)$, and the $\alpha$-energy of $\nu$ is controlled by (2.9), we derive that

$$
A_{2} \leq c_{9}\left(s_{0}^{\prime \prime}, s_{0}^{\prime}, n\right) \delta^{\min \left\{s_{0}^{\prime \prime}-1,1\right\}} \delta^{-\varepsilon_{1} / 2} \delta^{-\left(\varepsilon_{*}+\varepsilon_{0} n\right) / 2} .
$$

On the other hand, if we write

$$
A_{1}=\int \varphi_{c_{7}(n) \delta} * \varphi_{\eta} * \mu_{Q, e}(r) d \nu(r)
$$

and we recall that $\nu$ is supported in $H_{e, \delta, Q}:=\pi_{e}\left(L_{e, \delta, \text { bad, } Q}\right)$, then we can use the

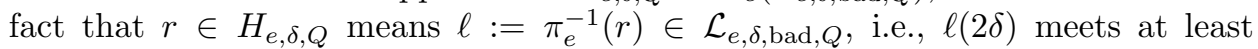
$\delta^{2 \varepsilon_{0}-1}$ dyadic cubes $R \in \mathcal{D}_{\delta}$ included in $Q$ which are not light, to deduce that $\mu_{Q}(\ell(2 \delta)) \geq \delta^{2 \varepsilon_{0}-1+n+\varepsilon_{*}}$ and, a fortiori,

$$
\varphi_{c_{7}(n) \delta} * \varphi_{\eta} * \mu_{Q, e}(r) \geq c_{10}(n) \delta^{2 \varepsilon_{0}+\varepsilon_{*}}
$$

for all $r \in H$ and $0<\eta \ll \delta$. Therefore,

$$
A_{1} \geq c_{10}(n) \delta^{2 \varepsilon_{0}+\varepsilon_{*}}
$$

because $\nu$ is a probability measure on $H$.

At this point, we get the desired contradiction $A_{1}>\left|A_{2}\right|$ for $\delta$ is sufficiently small because our choice (2.5) implies that $2 \varepsilon_{0}+\varepsilon_{*}<\min \left\{s_{0}^{\prime \prime}-1,1\right\}-\frac{\varepsilon_{1}}{2}-\frac{\varepsilon_{*}+\varepsilon_{0} n}{2}$.

2.6. End of the proof of Theorem 1.1. Let us take a decreasing sequence of dyadic scales $\delta_{j} \rightarrow 0$ such that $\delta_{j}^{\varepsilon_{0}}$ also a dyadic scale. We define the set $E_{\delta_{j}}$ of $\delta_{j}$-exceptional directions as

$$
E_{\delta_{j}}:=\bigcup_{Q \in \mathcal{D}_{\delta_{j}^{\varepsilon_{0}}}} E_{\delta_{j}, Q} .
$$

Since $\# \mathcal{D}_{\eta}=\eta^{-n}$, it follows from Lemma 2.4 that

$$
\mathcal{H}^{n-1}\left(E_{\delta_{j}}\right) \leq c_{2}\left(s_{0}^{\prime}, n\right) I_{s_{0}^{\prime}}(\mu) \delta_{j}^{\varepsilon_{1}-\varepsilon_{0} n} .
$$

Therefore, our choice of $\varepsilon_{1}>\varepsilon_{0} n$ in (2.4) implies

$$
\sum_{j=1}^{\infty} \mathcal{H}^{n-1}\left(E_{\delta_{j}}\right)<\infty
$$

so that the set

$$
E=E\left(s_{0}, s_{0}^{\prime}, s_{0}^{\prime \prime}, \varepsilon_{0}, \varepsilon_{1}, \varepsilon_{*}\right):=\bigcap_{n=1}^{\infty} \bigcup_{j \geq n} E_{\delta_{j}}
$$

has zero $\mathcal{H}^{n-1}$-measure.

We affirm that $\operatorname{dim}_{H}\left(\operatorname{Vis}_{e}(K)\right) \leq s_{0}$ whenever $e \in S^{n-1} \backslash E$. In fact, an element $e \notin E$ belongs to finitely many $E_{\delta_{j}}$ 's, say $e \notin E_{\delta_{j}}$ for all $j \geq j_{e}$.

By Lemma 2.3, we have $\mathcal{H}_{\infty}^{s_{0}}\left(\operatorname{Vis}_{e}(K) \cap K_{\delta_{j}, \text { light }}\right) \leq \mathcal{H}_{\infty}^{s_{0}}\left(K_{\delta_{j}, \text { light }}\right) \leq C(n) \cdot \delta_{j}^{\varepsilon_{*}}$ for all $j$. Also, by Lemma $2.5, \mathcal{H}_{\delta_{j}}^{s_{0}^{\prime}}\left(\operatorname{Vis}_{e}(K) \cap K_{\delta_{j} \text {, heavy }} \cap L_{e, \delta_{j}, \text { good }}\right) \leq \delta_{j}^{\varepsilon_{*}}$ for all $j$ sufficiently large. Moreover, $\mathcal{H}_{\infty}^{s_{0}^{\prime}}\left(\operatorname{Vis}_{e}(K) \cap K_{\delta_{j}, \text { heavy }} \cap \bigcup_{\substack{Q \in \mathcal{D}_{\delta_{j}^{\varepsilon_{0}}}, Q \cap K \neq \emptyset}} L_{e, \delta_{j}, \text { bad, } Q}\right) \leq$ $\delta_{j}^{\varepsilon_{*}}$ for all $j \geq j_{e}$ sufficiently large by Lemma 2.6 (and the fact that $\# \mathcal{D}_{\delta_{j}^{\varepsilon_{0}}}=\delta_{j}^{-\varepsilon_{0} n}$ ).

By putting these three estimates together, we derive that if $e \notin E$, then

$$
\mathcal{H}_{\infty}^{s_{0}}\left(\operatorname{Vis}_{e}(K)\right) \leq(C(n)+2) \delta_{j}^{\varepsilon_{*}}
$$


for all $j \geq j_{e}$ sufficiently large, and, consequently, $\operatorname{dim}_{H}\left(\operatorname{Vis}_{e}(K)\right) \leq s_{0}$ for all $e \notin E\left(s_{0}, s_{0}^{\prime}, s_{0}^{\prime \prime}, \varepsilon_{0}, \varepsilon_{1}, \varepsilon_{*}\right)$.

Since $s_{0}, s_{0}^{\prime}, s_{0}^{\prime \prime}, \varepsilon_{0}, \varepsilon_{1}, \varepsilon_{*}$ are arbitrary rational parameters satisfying (2.2), (2.3), (2.4) and (2.5), we conclude that

$$
\operatorname{dim}_{H}\left(\operatorname{Vis}_{e}(K)\right) \leq n-\min \left\{\frac{1}{5}, \frac{1}{n+2}\right\}
$$

for Lebesgue almost every $e \in S^{n-1}$.

\section{TyPiCAL Visible PARTS OF DYNAMicAl CANTOR SETS}

In this section, we revisit Orponen's method described above in order to establish Theorem 1.2.

3.1. Some preliminaries. It is well-known (see, e.g., [6] and [3]) that the products of $C^{2}$-dynamically defined Cantor sets of the real line and the self-similar sets given by a finite collection of Euclidean similarities verifying the open set condition defined a class of compact subsets $K \subset \mathbb{R}^{n}$ with the following properties:

- $K$ supports a measure $\mu$ equivalent to $\left.\mathcal{H}^{d}\right|_{K}, d:=\operatorname{dim}_{H}(K)$, such that $C^{-1} r^{d} \leq \mu(B(x, r)) \leq C r^{d}$ for all $x \in K, r>0$;

- there exists $\lambda>1$ such that, for all $\rho>0, K$ can be covered by a collection $\mathcal{C}_{\rho}(K)$ of disjoint cubes with sizes belonging to the interval $[\rho, \lambda \rho]$ such that their mutual distances are at least $\lambda^{-1} \rho$ and each of them contain a ball of radius $\lambda^{-1} \rho$ about some point of $K$.

In the context of Theorem 1.2, recall that we are also assuming that

$$
n \geq d>\max \left\{\sqrt{3}, \frac{(n-1)+\sqrt{(n-1)(n+3)}}{2}\right\} .
$$

Furthermore, up to rescaling, we can suppose that $K \subset[0,1]^{n}$.

Let us now fix some rational parameters

$$
\begin{gathered}
\max \left\{\frac{3 d+3}{d+3}, \frac{(n+1) d+(n-1)}{d+2}\right\}<n-\varepsilon_{0}<s_{0}^{\prime \prime}<s_{0}^{\prime}<s_{0}<d \leq n, \\
\alpha:=\min \left\{s_{0}^{\prime \prime}-1,2-\frac{s_{0}^{\prime}-(n-1)}{2}\right\} \\
\frac{\varepsilon_{0} d}{2}<\frac{\varepsilon_{1}}{2}<\min \left\{s_{0}^{\prime \prime}-1,1\right\}-\frac{\varepsilon_{0} d}{2}-2 \varepsilon_{0}-d+n,
\end{gathered}
$$

and

$$
0<\varepsilon_{*}<\min \left\{s_{0}^{\prime}+\varepsilon_{0}-n, 2\left(\min \left\{s_{0}^{\prime \prime}-1,1\right\}-\frac{\varepsilon_{0} d}{2}-2 \varepsilon_{0}-d+n-\frac{\varepsilon_{1}}{2}\right)\right\} .
$$

Note that these conditions are mutually compatible: indeed, our assumption (3.10) allows us to choose $s_{0}, s_{0}^{\prime}, s_{0}^{\prime \prime}$ and $\varepsilon_{0}$ in (3.11); since $\varepsilon_{0}<\min \left\{\frac{3-d}{d+3}, \frac{n-d+1}{d+2}\right\}$ and $s_{0}^{\prime}>n-\varepsilon_{0}$, we can select $\varepsilon_{1}$ in (2.4) and $\varepsilon_{*}$ in (2.5).

In what follows, $\delta=2^{-N}, N \in \mathbb{N}$, is an arbitrary (small) dyadic scale such that $\delta^{\varepsilon_{0}}$ is also a dyadic scale.

Our plan is to show Theorem 1.2 by following the same arguments from the previous section after some adjustments in the definitions and arguments. 
3.2. Absence of light cubes. In comparison with the previous section, our current setting is technically easier because there are no $\delta$-light cubes in the sense that any $Q \in \mathcal{C}_{\delta}(K)$ satisfies

$$
\mu(Q) \geq C^{-1} \lambda^{-d} \delta^{d}=: c_{11} \delta^{d} .
$$

3.3. Exceptional directions. Given a cube $Q \in \mathcal{C}_{\delta^{\varepsilon_{0}}}(K)$, we define

$$
E_{\delta, Q}:=\left\{e \in S^{n-1}: \int_{e^{\perp}}\left|\widehat{\mu_{Q}}(\zeta)\right|^{2} \cdot|\zeta|^{s_{0}^{\prime}-(n-1)} d \mathcal{H}^{n-1}(\zeta) \geq \delta^{-\varepsilon_{1}}\right\}
$$

where $\mu_{Q}=\left.\mu\right|_{Q}$. Since $s_{0}^{\prime}<d$, we have that

$$
\mathcal{H}^{n-1}\left(E_{\delta, Q}\right) \leq c_{2}\left(s_{0}^{\prime}, n\right) I_{s_{0}^{\prime}}(\mu) \delta^{\varepsilon_{1}}
$$

for all $Q \in \mathcal{C}_{\delta^{\varepsilon_{0}}}(K)$.

3.4. Good and bad lines. Denote by $\mathcal{L}_{e}$ the space of lines parallel to $e \in S^{n-1}$. Given a cube $Q \in \mathcal{C}_{\delta^{\varepsilon_{0}}}(K)$, the set $\mathcal{L}_{e, \delta, \text { bad, } Q}$ of $\delta$-bad lines in direction $e$ associated to $Q$ consists of all lines $\ell \in \mathcal{L}_{e}$ disjoint from $K \cap Q$ whose $2 \delta$-neighborhood $\ell(2 \delta)$ satisfy

$$
\#\left\{R \in \mathcal{C}_{\delta}(K): R \cap Q \neq \emptyset, R \cap \ell(2 \delta) \neq \emptyset\right\} \geq \delta^{2 \varepsilon_{0}-1} .
$$

We say that $\ell \in \mathcal{L}_{e}$ is a $\delta$-good line in the direction $e$ whenever $\ell \notin \mathcal{L}_{e, \delta \text {,bad, } Q}$ for all $Q \in \mathcal{C}_{\delta^{\varepsilon_{0}}}(K)$. The collection of $\delta$-good lines in the direction $e$ is denoted by $\mathcal{L}_{e, \delta \text {,good }}$ and we define

$$
L_{e, \delta, \operatorname{good}}:=\bigcup_{\ell \in \mathcal{L}_{e, \delta, \operatorname{good}}} \ell .
$$

Lemma 3.1. $\mathcal{H}_{\lambda \delta}^{s_{0}^{\prime}}\left(\operatorname{Vis}_{e}(K) \cap L_{e, \delta, \text { good }}\right) \leq \delta^{\varepsilon_{*}}$ for all $\delta$ sufficiently small.

Proof. The argument below is parallel to the proof of Lemma 2.5 above. Once again, let $\mathcal{T}_{e, \delta}$ be a collection of tubes of width $\delta$ whose bases are perpendicular to $e$ in order to cover $[0,1]^{n}$, so that our task is reduced to prove that, for each $T \in \mathcal{T}_{e, \delta}$, the minimal number $N\left(\operatorname{Vis}_{e}(K) \cap L_{e, \delta \text {,good }} \cap T, \delta\right)$ of balls of radii in the interval $[\delta, \lambda \delta]$ needed to cover $\operatorname{Vis}_{e}(K) \cap L_{e, \delta, \operatorname{good}} \cap T$ is at most

$$
N\left(\operatorname{Vis}_{e}(K) \cap L_{e, \delta, \operatorname{good}} \cap T, \delta\right) \leq c_{5}(n) \delta^{\varepsilon_{0}-1} .
$$

In order to estimate $N\left(\operatorname{Vis}_{e}(K) \cap L_{e, \delta \text {,good }} \cap T, \delta\right)$ for a given $T \in \mathcal{T}_{e, \delta}$, we consider two scenarios:

(i) for all $Q \in \mathcal{C}_{\delta^{\varepsilon_{0}}}(K)$, one has

$$
\#\left\{R \in \mathcal{C}_{\delta}(K): R \cap Q \neq \emptyset, R \cap T \neq \emptyset\right\}<\delta^{2 \varepsilon_{0}-1} ;
$$

(ii) there exists $Q_{1} \in \mathcal{C}_{\delta^{\varepsilon_{0}}}(K)$ with

$$
\#\left\{R \in \mathcal{C}_{\delta}(K): R \cap Q_{1} \neq \emptyset, R \cap T \neq \emptyset\right\} \geq \delta^{2 \varepsilon_{0}-1} .
$$

In the first scenario, we have that $N\left(\operatorname{Vis}_{e}(K) \cap L_{e, \delta \text {,good }} \cap T, \delta\right) \leq \delta^{\varepsilon_{0}-1}$ simply because $T$ can meet at most $\delta^{-\varepsilon_{0}}$ cubes $Q \in \mathcal{C}_{\delta^{\varepsilon_{0}}}(K)$.

In the second scenario, we take $Q_{1}$ to be a $\leq_{e}$-minimal cube with the property described in (ii) (in the sense that $Q_{1}$ minimizes $\inf \left\{\langle x, e\rangle: x \in Q_{1}\right\}$ among all cubes in (ii)). Since the $2 \delta$-neighborhood of any line $\ell \subset T$ contains $T$, we also have

$$
\#\left\{R \in \mathcal{C}_{\delta}(K): R \cap Q_{1}, R \cap \ell(2 \delta) \neq \emptyset\right\} \geq \delta^{2 \varepsilon_{0}-1} .
$$

Therefore, it follows from the definition of $\delta$-good line that any $\ell \in \mathcal{L}_{e, \delta \text {,good }}$ included in $T$ must intersect $K \cap Q_{1}$.

We affirm that

$$
\operatorname{Vis}_{e}(K) \cap L_{e, \delta, \operatorname{good}} \cap T \cap Q=\emptyset
$$


for any cube $Q \in \mathcal{C}_{\delta^{\varepsilon_{0}}}(K)$ with $\inf \{\langle x, e\rangle: x \in Q\}>\sup \left\{\langle y, e\rangle: y \in Q_{1}\right\}$. In fact, if $x \in \operatorname{Vis}_{e}(K) \cap L_{e, \delta \text {,good }} \cap T \cap Q$, then $\pi_{e}(x)=\pi_{e}(y)$ for some $y \in Q_{1}$. Since $\langle x, e\rangle>\langle y, e\rangle$, one would get $x \notin \operatorname{Vis}_{e}(K)$, a contradiction.

Hence, $\operatorname{Vis}_{e}(K) \cap L_{e, \delta \text {,good }} \cap T$ is covered by the collection of cubes $Q \in \mathcal{C}_{\delta^{\varepsilon_{0}}}(K)$ with $\inf \{\langle x, e\rangle: x \in Q\} \leq \sup \left\{\langle y, e\rangle: y \in Q_{1}\right\}$. Now, we observe that

- the number of cubes $Q \in \mathcal{C}_{\delta^{\varepsilon_{0}}}(K)$ intersecting $T$ with

$$
\inf \left\{\langle z, e\rangle: z \in Q_{1}\right\} \leq \inf \{\langle x, e\rangle: x \in Q\} \leq \sup \left\{\langle y, e\rangle: y \in Q_{1}\right\}
$$

is bounded by an absolute constant $c_{4}(n)$; for each of them, we will use the crude bound $N\left(\operatorname{Vis}_{e}(K) \cap L_{e, \delta \text {,good }} \cap T, \delta\right) \leq \delta^{\varepsilon_{0}-1}$ coming from the fact that $Q \cap T$ can be covered using at most $\delta^{\varepsilon_{0}-1}$ balls of radius $\delta$;

- any cube $Q \in \mathcal{C}_{\delta^{\varepsilon_{0}}}(K)$ intersecting $T \cap K$ with

$$
\inf \left\{\langle z, e\rangle: z \in Q_{1}\right\}>\inf \{\langle x, e\rangle: x \in Q\}
$$

satisfies

$$
\#\left\{R \in \mathcal{C}_{\delta}(K): R \cap Q_{1} \neq \emptyset, R \cap \ell(2 \delta) \neq \emptyset\right\} \leq \delta^{2 \varepsilon_{0}-1}
$$

because of the $\leq_{e}$-minimality of $Q_{1}$; the number of such cubes $Q$ is at most $\leq \delta^{-\varepsilon_{0}}$ because $T$ meets at most $\delta^{-\varepsilon_{0}}$ cubes $Q \in \mathcal{C}_{\delta^{\varepsilon_{0}}}(K)$.

By combining the estimates above, we conclude that

$$
N\left(\operatorname{Vis}_{e}(K) \cap L_{e, \delta, \operatorname{good}} \cap T, \delta\right) \leq c_{4}(n) \delta^{\varepsilon_{0}-1}+\delta^{-\varepsilon_{0}} \delta^{2 \varepsilon_{0}-1}=c_{5}(n) \delta^{\varepsilon_{0}-1} .
$$

This completes the proof.

3.5. Typical visible parts in bad lines. Similarly to the previous section, the last step towards the proof of Theorem 1.2 is the following estimate:

Lemma 3.2. Let $Q \in \mathcal{C}_{\delta^{\varepsilon_{0}}}(K)$ be a cube, consider a direction e $\notin E_{\delta, Q}$, and denote $L_{e, \delta, b a d, Q}:=\bigcup_{\ell \in \mathcal{L}_{e, \delta, b a d, Q}} \ell$. Then,

$$
\mathcal{H}_{\infty}^{s_{0}^{\prime}-1}\left(\pi_{e}\left(L_{e, \delta, b a d, Q}\right)\right) \leq \delta^{\varepsilon_{*}+\varepsilon_{0} d}
$$

for all $\delta$ sufficiently small.

Proof. By contradiction, suppose that $\mathcal{H}_{\infty}^{s_{0}^{\prime}-1}\left(\pi_{e}\left(L_{e, \delta \text {,bad }, Q}\right)\right) \geq \delta^{\varepsilon_{*}+\varepsilon_{0} d}$. By Lemma 2.1 , we have a probability measure $\nu$ supported on $H_{e, \delta, Q}:=\pi_{e}\left(L_{e, \delta, \text { bad,Q }}\right)$ with

$$
\nu(B(x, r)) \leq C(n-1) \delta^{-\varepsilon_{*}-\varepsilon_{0} d} r^{s_{0}^{\prime}-1}
$$

for all $x \in H$ and $r>0$. Thus, our choice of $\alpha \leq s_{0}^{\prime \prime}-1<s_{0}^{\prime}-1$ in (3.12) (and Remark 2.2) means that the $\alpha$-energy of $\nu$ satisfies

$$
c_{1}(\alpha, n-1) \int|\widehat{\nu}(\xi)|^{2} \cdot|\xi|^{\alpha} d \xi=I_{\alpha}(\nu) \leq c_{6}\left(s_{0}^{\prime \prime}, s_{0}^{\prime}, n\right) \delta^{-\varepsilon_{*}-\varepsilon_{0} d} .
$$

Next, we observe that, by definition, any line $\ell \in \mathcal{L}_{e, \delta \text {,bad, } Q}$ misses $K \cap Q$. Therefore, $\mu_{Q, e}:=\left(\pi_{e}\right)_{*}\left(\mu_{Q}\right)$ and $\nu$ have disjoint supports. Hence, if we fix a non-negative smooth bump function $\varphi$ on $e^{\perp} \simeq \mathbb{R}^{n-1}$ with total integral one and $\varphi(0)=1$, then

$$
\begin{aligned}
0 & =\int \varphi_{\eta} * \mu_{Q, e} d \nu=\int \widehat{\varphi}(\eta \xi) \widehat{\mu_{Q, e}}(\xi) \overline{\widehat{\nu}(\xi)} d \xi \\
& =\int\left(1-\widehat{\varphi}\left(c_{7}(n) \delta \xi\right)\right) \widehat{\varphi}(\eta \xi) \widehat{\mu_{Q, e}}(\xi) \overline{\widehat{\nu}(\xi)} d \xi+\int \widehat{\varphi}\left(c_{7}(n) \delta \xi\right) \widehat{\varphi}(\eta \xi) \widehat{\mu_{Q, e}}(\xi) \overline{\widehat{\nu}(\xi)} d \xi \\
& :=A_{2}-A_{1}
\end{aligned}
$$

for all $0<\eta \ll \delta$, where $\varphi_{\eta}(x)=\varphi(\eta x) / \eta^{n-1}$. 
Once more, we will reach a contradiction with the identity in the previous paragraph by showing that $\left|A_{2}\right|<\left|A_{1}\right|$. For this sake, we observe that $\widehat{\varphi}$ is a bounded Lipschitz function with $\widehat{\varphi}(0)=1$, so that $\left|1-\widehat{\varphi}\left(c_{7}(n) \delta \xi\right)\right| \leq c_{8}(n) \delta|\xi|$ and, a fortiori,

$$
\left|A_{2}\right| \leq c_{8}(n) \delta^{\frac{s_{0}^{\prime}-(n-1)}{2}+\frac{\alpha}{2}}\left(\int\left|\widehat{\mu_{Q, e}}(\xi)\right|^{2} \cdot|\xi|^{s_{0}^{\prime}-(n-1)} d \xi\right)^{1 / 2}\left(\int|\widehat{\nu}(\xi)|^{2} \cdot|\xi|^{\alpha} d \xi\right)^{1 / 2}
$$

thanks to our choice of $\frac{s_{0}^{\prime}-(n-1)}{2}+\frac{\alpha}{2} \leq 1$ in (3.12) and the Cauchy-Schwarz inequality. By plugging into the previous inequality the facts that our choices in (3.11) and (3.12) imply $\frac{s_{0}^{\prime}-(n-1)}{2}+\frac{\alpha}{2} \geq \min \left\{s_{0}^{\prime \prime}-1,1\right\}$, our assumption $e \notin E_{\delta, Q}$ allows (by definition) to control $\left|\widehat{\mu_{Q}}(\xi)\right|\left(=\left|\widehat{\mu_{Q, e}}(\xi)\right|\right.$ for $\left.\xi \in e^{\perp}\right)$, and the $\alpha$-energy of $\nu$ is controlled by (3.17), we derive that

$$
A_{2} \leq c_{9}\left(s_{0}^{\prime \prime}, s_{0}^{\prime}, n\right) \delta^{\min \left\{s_{0}^{\prime \prime}-1,1\right\}} \delta^{-\varepsilon_{1} / 2} \delta^{-\left(\varepsilon_{*}+\varepsilon_{0} d\right) / 2} .
$$

On the other hand, if we write

$$
A_{1}=\int \varphi_{c_{7}(n) \delta} * \varphi_{\eta} * \mu_{Q, e}(r) d \nu(r)
$$

and we recall that $\nu$ is supported in $H_{e, \delta, Q}:=\pi_{e}\left(L_{e, \delta \text {,bad,Q }}\right)$, then we can use the fact that $r \in H_{e, \delta, Q}$ means $\ell:=\pi_{e}^{-1}(r) \in \mathcal{L}_{e, \delta \text {,bad, } Q}$, i.e., $\ell(2 \delta)$ meets at least $\delta^{2 \varepsilon_{0}-1}$ cubes $R \in \mathcal{C}_{\delta}(K)$ intersecting $Q$ and verifying (3.15), to deduce that $\mu_{Q}(\ell(2 \delta)) \geq$ $c_{11} \delta^{2 \varepsilon_{0}-1+d}$ and, a fortiori,

$$
\varphi_{c_{7}(n) \delta} * \varphi_{\eta} * \mu_{Q, e}(r) \geq c_{11} c_{10}(n) \delta^{2 \varepsilon_{0}-1+d-(n-1)}
$$

for all $r \in H$ and $0<\eta \ll \delta$. Therefore,

$$
A_{1} \geq c_{11} c_{10}(n) \delta^{2 \varepsilon_{0}+d-n}
$$

because $\nu$ is a probability measure on $H$.

At this point, we get the desired contradiction $A_{1}>\left|A_{2}\right|$ for $\delta$ is sufficiently small because our choice (3.14) implies that $2 \varepsilon_{0}+d-n<\min \left\{s_{0}^{\prime \prime}-1,1\right\}-\frac{\varepsilon_{1}}{2}-\frac{\varepsilon_{*}+\varepsilon_{0} d}{2}$.

3.6. End of the proof of Theorem 1.2. Let us take a decreasing sequence of dyadic scales $\delta_{j} \rightarrow 0$ such that $\delta_{j}^{\varepsilon_{0}}$ also a dyadic scale. We define the set $E_{\delta_{j}}$ of $\delta_{j}$-exceptional directions as

$$
E_{\delta_{j}}:=\bigcup_{Q \in \mathcal{C}_{\delta_{j}}(K)} E_{\delta_{j}, Q}
$$

Since $\# \mathcal{C}_{\eta}(K) \leq c_{12} \eta^{-d}$ (thanks to (3.15) and the finiteness of $\mu$ ), it follows from (3.16) that

$$
\mathcal{H}^{n-1}\left(E_{\delta_{j}}\right) \leq c_{2}\left(s_{0}^{\prime}, n\right) I_{s_{0}^{\prime}}(\mu) \delta_{j}^{\varepsilon_{1}-\varepsilon_{0} d}
$$

Therefore, our choice of $\varepsilon_{1}>\varepsilon_{0} d$ in (3.13) implies

$$
\sum_{j=1}^{\infty} \mathcal{H}^{n-1}\left(E_{\delta_{j}}\right)<\infty
$$

so that the set

$$
E=E\left(s_{0}, s_{0}^{\prime}, s_{0}^{\prime \prime}, \varepsilon_{0}, \varepsilon_{1}, \varepsilon_{*}\right):=\bigcap_{n=1}^{\infty} \bigcup_{j \geq n} E_{\delta_{j}}
$$

has zero $\mathcal{H}^{n-1}$-measure.

We affirm that $\operatorname{dim}_{H}\left(\operatorname{Vis}_{e}(K)\right) \leq s_{0}^{\prime}$ whenever $e \in S^{n-1} \backslash E$. In fact, an element $e \notin E$ belongs to finitely many $E_{\delta_{j}}$ 's, say $e \notin E_{\delta_{j}}$ for all $j \geq j_{e}$. 
By Lemma 3.1, $\mathcal{H}_{\lambda \delta_{j}}^{s_{0}^{\prime}}\left(\operatorname{Vis}_{e}(K) \cap L_{e, \delta_{j}, \text { good }}\right) \leq \delta_{j}^{\varepsilon_{*}}$ for all $j$ sufficiently large. Moreover, $\mathcal{H}_{\infty}^{s_{0}^{\prime}}\left(\operatorname{Vis}_{e}(K) \cap \bigcup_{Q \in \mathcal{C}_{\delta_{j} \varepsilon_{0}}(K)} L_{e, \delta_{j}, \text { bad }, Q}\right) \leq c_{12} \delta_{j}^{\varepsilon_{*}}$ for all $j \geq j_{e}$ sufficiently large by Lemma 3.2 (and the fact that $\# \mathcal{C}_{\delta_{j}^{\varepsilon_{0}}}(K) \leq c_{12} \delta_{j}^{-\varepsilon_{0} d}$ ).

By putting these three estimates together, we derive that if $e \notin E$, then

$$
\mathcal{H}_{\infty}^{s_{0}^{\prime}}\left(\operatorname{Vis}_{e}(K)\right) \leq\left(c_{12}+1\right) \delta_{j}^{\varepsilon_{*}}
$$

for all $j \geq j_{e}$ sufficiently large, and, consequently, $\operatorname{dim}_{H}\left(\operatorname{Vis}_{e}(K)\right) \leq s_{0}^{\prime}$ for all $e \notin E\left(s_{0}, s_{0}^{\prime}, s_{0}^{\prime \prime}, \varepsilon_{0}, \varepsilon_{1}, \varepsilon_{*}\right)$.

Since $s_{0}, s_{0}^{\prime}, s_{0}^{\prime \prime}, \varepsilon_{0}, \varepsilon_{1}, \varepsilon_{*}$ are arbitrary rational parameters satisfying (3.11), (3.12), (3.13) and (3.14), we conclude that

$$
\operatorname{dim}_{H}\left(\operatorname{Vis}_{e}(K)\right) \leq \max \left\{\frac{3 d+3}{d+3}, \frac{(n+1) d+(n-1)}{d+2}\right\}
$$

for Lebesgue almost every $e \in S^{n-1}$.

\section{REFERENCES}

[1] I. Arhosalo, E. Järvenpää, M. Järvenpää, M. Rams, P. Shmerkin, Visible parts of fractal percolation, Proc. Edinb. Math. Soc. (2) 55 (2012), 311-331.

[2] K. Falconer, J. Fraser, The visible part of plane self-similar sets, Proc. Amer. Math. Soc. 141 (2013), 269-278.

[3] J. Hutchinson, Fractals and self-similarity, Indiana Univ. Math. J. 30 (1981), 713-747.

[4] E. Järvenpää, M. Järvenpää, P. MacManus, T. O'Neil, Visible parts and dimensions, Nonlinearity, 16 (2003), 803-818.

[5] E. Järvenpää, M. Järvenpää, J. Niemelä, Transversal mappings between manifolds and nontrivial measures on visible parts, Real Anal. Exchange 30 (2004/05), no. 2, 675-687.

[6] Y. Lima, C. Moreira, A combinatorial proof of Marstrand's theorem for products of regular Cantor sets, Expo. Math. 29 (2011), 231-239.

[7] J. Marstrand, Some fundamental geometrical properties of plane sets of fractional dimensions, Proc. London Math. Soc. (3) 4, (1954), 257-302.

[8] P. Mattila, Hausdorff dimension, projections, and the Fourier transform, Publ. Mat. 48 (2004), 3-48.

[9] T. Orponen, On the dimension of visible parts, preprint (2019) available at arXiv:1912.10898.

Carlos Matheus

CMls, CNRS, École polytechnique, Institut Polytechnique de Paris, 91128

Palaiseau Cedex, France.

E-mail address: carlos.matheus@math.cnrs.fr 